\title{
ANFIS Inverse Kinematics and Hybrid Control of a Human Leg Gait Model
}

\author{
${ }^{1}$ Arif Ankarali, ${ }^{2}$ Murat Cilli \\ ${ }^{1}$ Faculty of Aeronautics and Astronautics, Department of Aeronautical Engineering, N. Erbakan University Turkey \\ ${ }^{2}$ School of Physical Education and Sports, Sakarya University Turkey \\ *Corresponding author: Address: Faculty of Aeronautics and Astronautics, Department of Aeronautical Engineering, \\ N. Erbakan University, Konya, TURKEY. E-mail address: ankaraliarif@gmail.com, Phone: +903323238220 Fax: \\ $+903322362140$
}

\begin{abstract}
A hybrid learning procedure referred to as adaptive neuro fuzzy inference system (ANFIS) is applied to an artificial leg model to generate the correct positions of the servomotors actuating the leg joints. One of the most important control problems of mechanical arms and legs is the efficient calculation of correct joint angles for a space trajectory. Although this application represents the simplest model with two degrees of freedom, the practicality of ANFIS for such mechanical systems is validated. For the gait model of the proposed mechanism, the experimental planar motion of the ankle joint is transformed to joint angles by ANFIS and approximated by polynomial functions. The corresponding servomotor positions are obtained by the proposed inverse kinematic solution method and are included in a Simulink model as an embedded Matlab function. A hybrid control system consisting of combination of a proportional plus derivative (PD) controller and a fuzzy logic controller (FLC) is applied to control the selected servomotors. The accuracy of the control system is further verified on SimMechanics.
\end{abstract}

Keywords: ANFIS; inverse kinematics; Fuzzy logic; Hybrid control; Gait model

\section{Introduction}

Simulation of robotic systems is made easier because of the development in modeling and simulation tools. The original capabilities of Simulink have been extended by SimMechanics to further improve the modeling of real time systems. The simulation based any control system can be designed and validated, and the generated control signals can be applied directly to the Simulink/SimMechanics model of the existing real system. By means of some complementary embedded Matlab functions, any sub system may be added to the simulation in order to improve the outset of the Simulink model. This enables researchers, designers and manufactures to improve the quality of their end products as discussed in detail in [1]. The development of today's humanoid robots and artificial limbs represent a popular research area of science.
In particular, inverse kinematics of open loop mechanical system represents an interesting subject due to its nonlinear nature. There exist many different approaches to create an efficient method to calculate the joint parameters for known positions and orientations of the robot. Mostly commonly, the Denavit-Hartanberg (D$\mathrm{H})$ approach and the geometric approach are prevalent and are both described in details in $[2,3]$. Qiao et al. [4] further solved the inverse kinematics problem of a general $6 \mathrm{R}$ robot by using double quaternions and Dixon resultant. Dinesh Manocha and John F. Canny [5] developed an algorithm that transforms the inverse kinematics problem of a $6 \mathrm{R}$ manipulator to the solution of a polynomial expression. Another approach proposes to solve the inverse position kinematics problem of manipulators with the double universal joint wrist, and a numerical singularity 
analysis is presented for such manipulators [6]. By utilizing the easier-to-use direct kinematic solution of the manipulator, one can derive the inverse solutions with ANFIS [7]. Basically, the solution involves the training of a system by using the obtained information by direct kinematics and constructing relevant lookup tables. The details of ANFIS system is discussed by Jyh-Shing Roger Jang [8]. Design, development and implementation of FLC for servomotor controls is another interesting area in these studies. The approach of rapid prototyping may be used for this purpose and the output of the control signals may drive a real servomotor to accomplish a preplanned motion. The experimentally implemented study on the SCARA robot control by Er et al. and Popercu et al. represent good examples for such studies $[9,10]$.

In this paper, a traditional prosthetic leg is considered. The device consists of a custom fitted socket, an internal structure as well as knee cuffs and belts that attach to the body, all of which are shown in Fig. 1(a).

It is assumed that there exist two actuated joints that enable the leg to deliver gait patterns. One of the actuators is located at the hip while the other one is located at the knee. The angular motions of these joints are applied by means of two permanent-magnet (PM) servomotors with gearboxes.
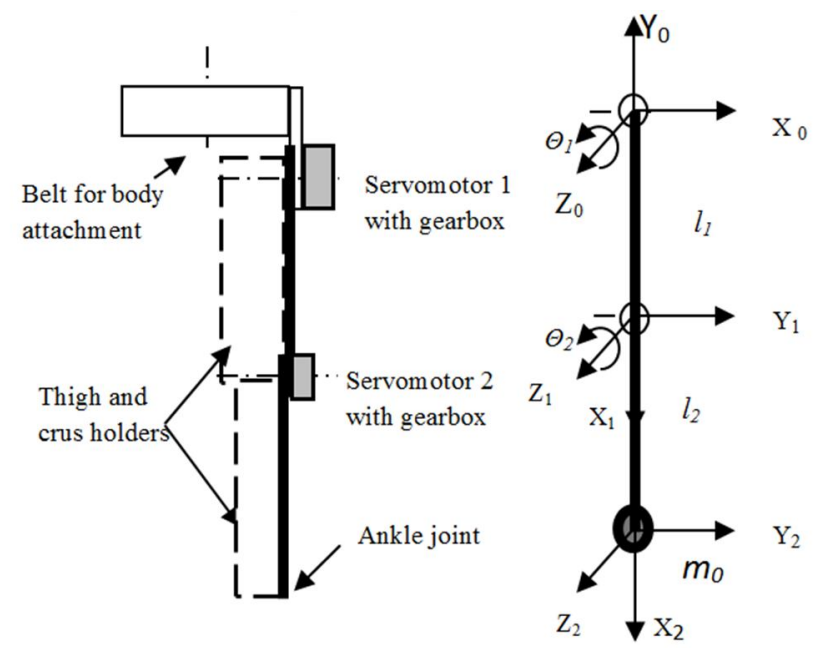

(b)

Figure 1. Simplified model of the servomotor driven artificial limb.

The first servomotor is rigidly connected to the belt and spins the thigh around its joint axis, which generates the hip motion. The second servomotor gives the knee its rotation and is assembled at the extension of the upper part where it rotates the crust of the leg to complete the walking cycle. Here, the $x-y$ plane moment effect of the leg is not taken into consideration, which is a design parameter of the leg holder mechanism. The simplified model of the mentioned system is given in Fig. 1(b). The servomotors employed in this system are typically supplied with their own proportionalintegral- derivative (PID) controllers.

\section{Kinematic Equations of The Mechanism}

\subsection{Direct kinematic equations}

Although D-H approach is applicable to all robotic systems, the direct kinematic equations of this simple mechanism can be written by considering the geometry of the mechanism itself. In a matrix form, the kinematics of this system can be written as 
$\left[\begin{array}{l}x \\ y\end{array}\right]=\left[\begin{array}{cc}\cos \theta_{1} & \cos \left(\theta_{1}+\theta_{2}\right) \\ \sin \theta_{1} & \sin \left(\theta_{1}+\theta_{2}\right)\end{array}\right]\left[\begin{array}{l}l_{1} \\ l_{2}\end{array}\right]$

\subsection{Inverse Kinematic Equations}

The kinematic equations can be derived by analyzing Fig. 2. To avoid using the arccosine and arcsine functions, arctangent 2 identity can be used [2] as follows

$$
\cos \theta_{2}=\frac{x^{2}+y^{2}-l_{1}{ }^{2}-l_{2}{ }^{2}}{2 l_{1} l_{2}}
$$

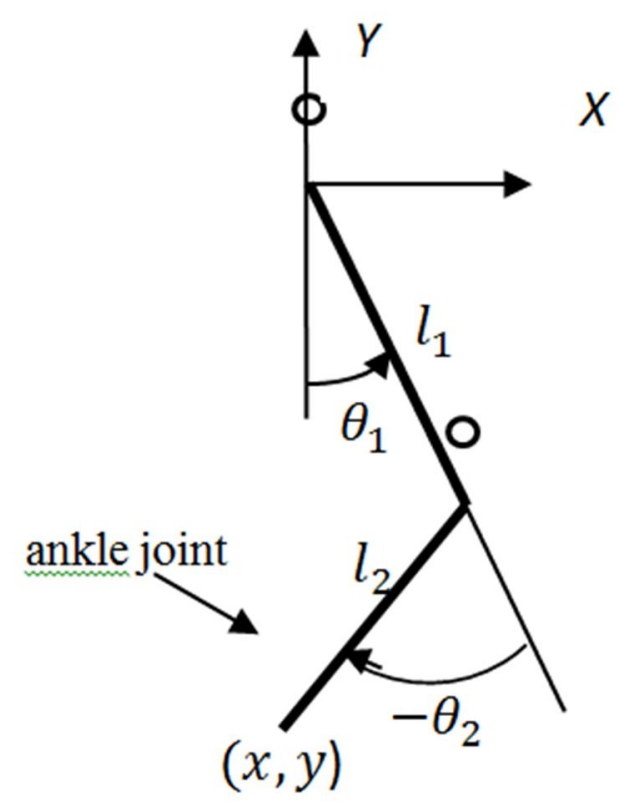

Figure 2. Ankle joint Cartesian position with angular joints.

$$
\begin{aligned}
& \sin \theta_{2}=-\sqrt{1-\cos ^{2} \theta_{2}} \\
& \theta_{2}=2 \pi+\operatorname{atan} 2\left(\sin \theta_{2}, \cos \theta_{2}\right)
\end{aligned}
$$

and

$$
\theta_{1}=\pi+\left(\operatorname{atan} 2(y, x)-\operatorname{atan} 2\left(l_{2} \sin \theta_{2}, l_{1+} l_{2} \cos \theta_{2}\right)\right)
$$

\section{Mathematical Model}

The proposed system consists of two legs and two joints with a tip payload as seen in Fig. 1. The mathematical model of this system can be derived through Lagrangian dynamics [2]. The Lagrangian of any system can be written as the difference between the kinetic and potential energies

$$
L=K-V
$$

The Lagrange equation of motion for this system can be derived by applying the Lagrange principle of conservation of energy

$$
\frac{d}{d t}\left(\frac{\partial L}{\partial \dot{q}_{l}}\right)-\left(\frac{\partial L}{\partial q_{i}}\right)=Q_{i}, \quad i=1,2
$$

The equations of motion for the system can be re-written more conveniently in a matrix format as follows

$$
D(q) \ddot{q}+C(q, \dot{q}) \dot{q}+G(q)=Q
$$

The elements of this matrix are given in the appendix. The mass and the length of the thigh is $m_{l l}$ and $l_{l}$; and the mass and the length of the crus is $m_{l 2}$ and $l_{2}$. The foot is assumed as a point mass $m_{0}$ representing the foot at the ankle joint. $\theta_{1}$ is the angular position of the hip joint and $\theta_{2}$ is the angular position of the knee joint provided by the servomotors with gears. 


\section{Control System Design}

The trajectory of a foot for a walking cycle is approximated by a time dependent polynomial. At each sampling time, the desired angular positions of the hip joint and the knee joint are calculated by ANFIS model. These angular positions are introduced to the control system as the desired joint angles that must be satisfied by the servomotors. The specifications of the servomotors and the gear train selected for the walking mechanism are as outlined in Table 1. The nominal torque information necessary to select the servomotors are obtained from the forward dynamics analysis that SimMechanics generated based on the simulation of the leg model. These torque specifications are verified by the experimental data given in [11] for one leg with a maximum torque of $50 \mathrm{Nm}$ and a length $l=0.87 \mathrm{~m}$ generating a $1.3 \mathrm{~m} . \mathrm{s}^{-1}$ walking velocity. The length of the thigh and the crus are selected as $0.46 \mathrm{~m}$ and $0.41 \mathrm{~m}$, respectively. The calculated moment of inertia using the expression $I=(1 / 3) m L^{2}$ are $0.70 \mathrm{~kg} \cdot \mathrm{m}^{2}$ and 0.22 $\mathrm{kg} . \mathrm{m}^{2}$ where $m_{1}$ is $10 \mathrm{~kg}$ and $m_{2}$ is $4 \mathrm{~kg}$. A $1 \mathrm{~kg}$ point mass is assumed at the ankle joint representing the foot mass $m_{0}$.

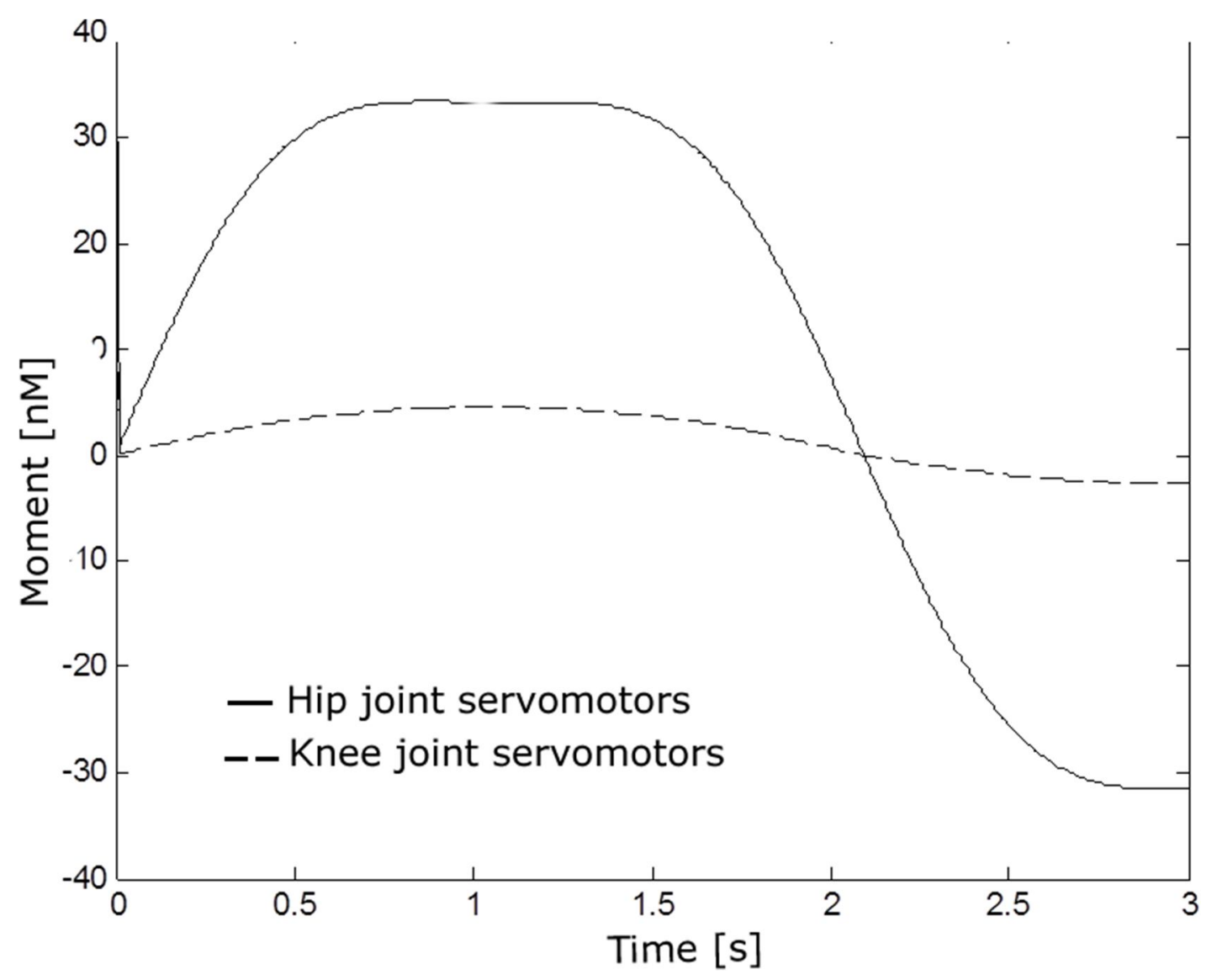

Figure 3. Hip and knee joint servomotors moment variations. 
Table 1. The specifications of the servomotors and the gear boxes.

\begin{tabular}{|l|c|c|}
\hline Motor Data & $\begin{array}{c}\text { Hip joint } \\
\text { Servomotor-Maxon }\end{array}$ & $\begin{array}{c}\text { Knee joint } \\
\text { Servomotor-Maxon }\end{array}$ \\
\hline Characteristics & & \\
\hline Terminal resistance $(\mathrm{Ohm})$ & 0.363 & 5.04 \\
\hline Terminal inductance $(\mathrm{mH})$ & 0.264 & 2.24 \\
\hline Torque constant $(\mathrm{mNm} / \mathrm{A})$ & 70.5 & 51 \\
\hline Speed constant $(\mathrm{rpm} / \mathrm{V})$ & 135 & 187 \\
\hline Mechanical time constant $(\mathrm{ms})$ & 22.3 & 92.5 \\
\hline Rotor inertia $\left(\mathrm{gcm}^{2}\right)$ & 3060 & $\begin{array}{c}\text { Knee joint Gear } \\
\end{array}$ \\
\hline Gearhead Data & Hip joint gear & $\begin{array}{c}\text { Planetary Gearhead GP } \\
42 \text { C ø42 mm, 3-15 }\end{array}$ \\
& Planetary Gearhead GP \\
& 52 C ø52 mm, 4-30 & Mm \\
\hline Reduction & Nm & $1296: 1$ \\
\hline Mass inertia $\left(\mathrm{gcm}^{2}\right)$ & Maxon- $(223111)$ & 5 \\
\hline Max. motor shaft diameter $(\mathrm{mm})$ & $756: 1$ & 4 \\
\hline
\end{tabular}

Table 2. Experimentally measured values of a gait model for one step.

\begin{tabular}{|c|c|c|c|c|c|c|c|c|c|c|c|c|}
\cline { 2 - 11 } \multicolumn{1}{c|}{} & 1 & 2 & 3 & 4 & 5 & 6 & 7 & 8 & 9 & 10 & 11 & 12 \\
\hline $\mathrm{X}(\mathrm{mm})$ & -34 & -31.6 & -29 & -25.5 & -18.5 & -2 & 2 & 20.5 & 26 & 29.5 & 32 & 34 \\
\hline $\mathrm{Y}(\mathrm{mm})$ & -800 & -770 & -740 & -710 & -680 & -650 & -650 & -680 & -710 & -740 & -770 & -800 \\
\hline
\end{tabular}

From Fig. 3, it is clear that the hip joint servomotor torque peaks at $35 \mathrm{Nm}$ and that the knee joint servomotor peaks at $5 \mathrm{Nm}$ for a frequency of $1.5 \mathrm{rad} / \mathrm{s}$ for the sinusoidal motion applied at each joint. For the model of the human gait, although all the motions of joints may be generated from camera captured data [12] the values for the ankle joint given in Table 2 were obtained by simply marking the positions where the hip joint is fixed. The trajectory of the ankle joint is calculated by means of polynomial curve fitting of the experimental data. It is assumed that the total time for one step is $2 \mathrm{~s}$ and that the time intervals between each measurement are equal. An acceptable $5^{\text {th }}$ order polynomial fit is applied to the experimental data as shown in Eqn. (9). The ankle joint trajectory and the simplified leg model are shown together in Fig. 4.

The polynomial function of the gait model is

$$
\begin{aligned}
& f(x)=\mathrm{p}_{1} x^{5}+\mathrm{p}_{2} x^{4}+\mathrm{p}_{3} x^{3}+\mathrm{p}_{4} x^{2}+ \\
& \mathrm{p}_{5} x+\mathrm{p}_{6}
\end{aligned}
$$




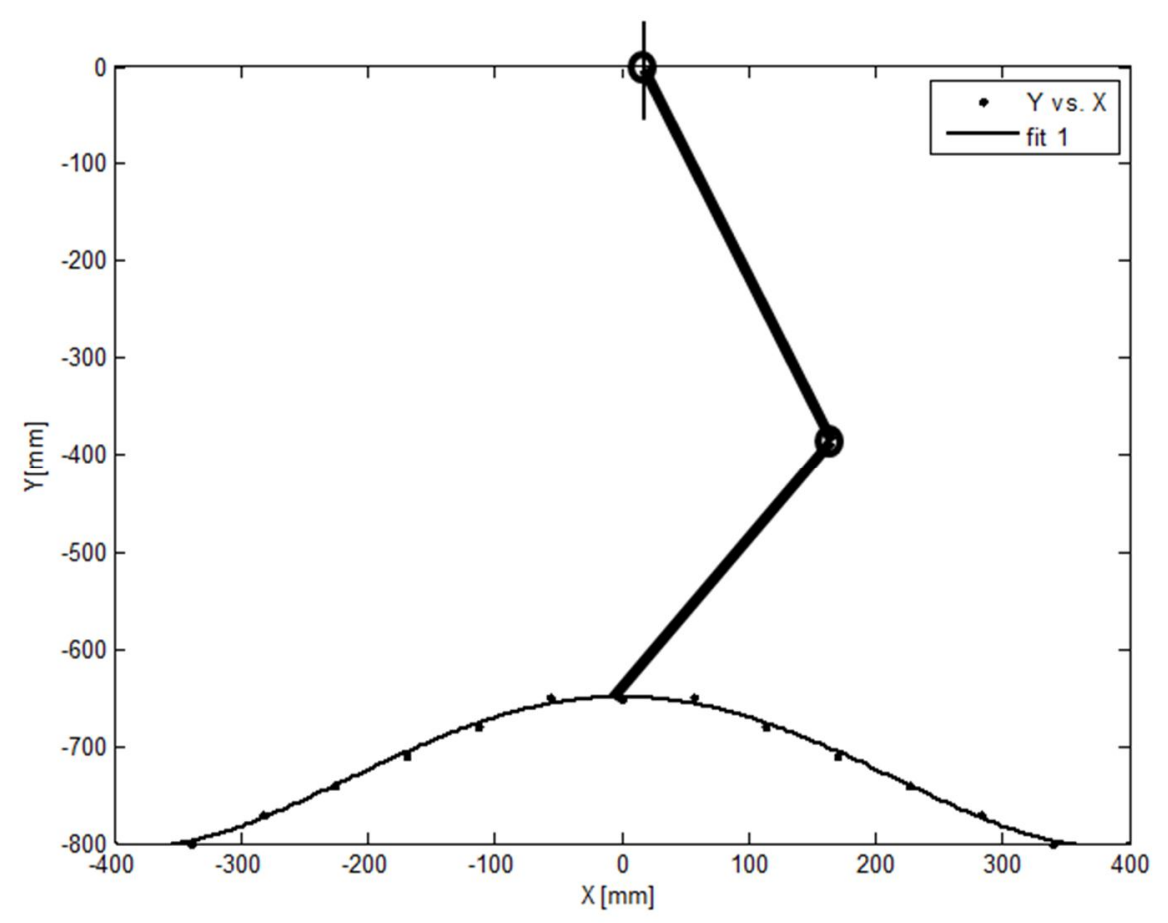

Figure. 4. Gait model with Cartesian ankle joint trajectory.

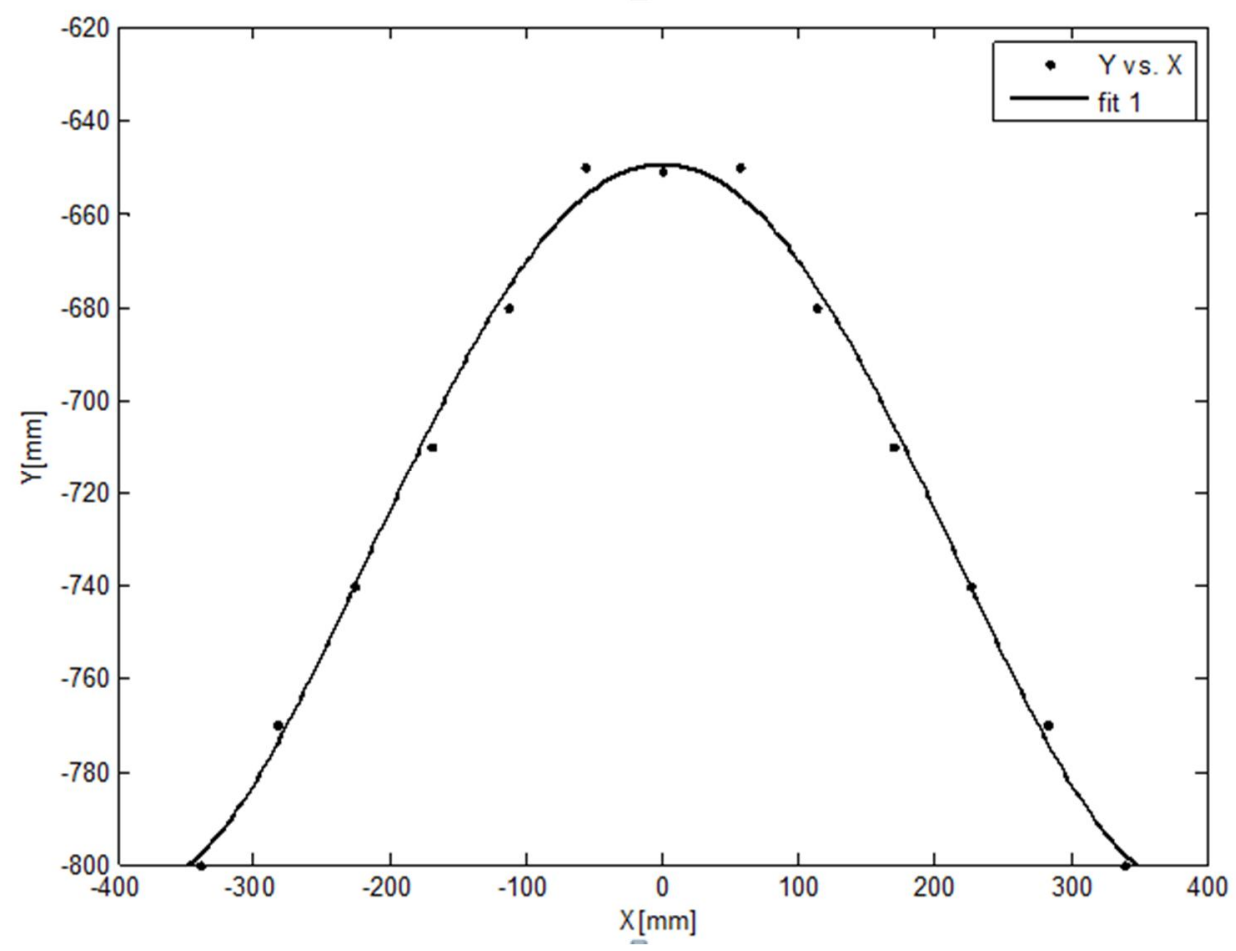

Figure 5. The ankle joint Cartesian trajectory of the gait model.

where the coefficients of the equation are $p_{1}=-$ $1.371 \mathrm{e}-025, \mathrm{p}_{2}=7.592 \mathrm{e}-009, \quad \mathrm{p}_{3}==-6.073 \mathrm{e}-$ $009, \mathrm{p}_{4}=-0.002164, \mathrm{p}_{5}=0.0008657$ and $\mathrm{p}_{6}=-$ 649.4

With this, the problem can now be reduced to the control of the selected servomotors such that the Cartesian trajectory of the ankle joint, as seen in Fig. 5, can be tracked precisely. In this study, the FLC plus PD control will be applied to the servomotors. There exist many control techniques that may be applied to improve the trajectory tracking performance $[13,14]$; however, hybrid control algorithms 
such as FLC plus PD controls are superior in most set-point regulator problems because of their adaptive nature $[15,16,17]$. FLC is applied to $\mathrm{PD}$ control in parallel, such that, an adaptive integrator part is included in the overall control system. At each sampling time, the FLC part of the control system is capable of producing a control signal based on the integral gain constant and in tandem with the changing dynamics of the leg mechanism. It must be understood that because the real system is modeled through SimMechanics, the effect of the inertial forces and coupling will be included in a different way as applied in computed torque techniques. The basic independent $j 40$ control diagram is shown in Fig. 6.

\section{ANFIS Modeling of The Workspace}

In robotic applications, direct kinematic analysis can be done by several methods and the position/orientation of the end effector can be calculated precisely. The direct kinematic analysis gives the exact position and orientation of a robotic manipulator which generates a very useful database for inverse kinematic approximations. These can be used with "ANFIS" Matlab tools to train the fuzzy inference system (FIS) model membership functions based on the acceptable error criterion.

For real world applications, inverse kinematic analysis is generally required. Such analysis may have a varying degree of complexity that depends on the nonlinearity of the governing equations. To overcome this difficulty, ANFIS network can be applied to get the approximate joint coordinates with a good accuracy. The application of this method reduces the complexity of the nonlinear mathematical equations and the difficulties of their solutions. In this modeling technique, the data obtained through the direct kinematic solution is employed to create the adaptive neural network system and to train the membership functions of the fuzzy inference system (FIS) similar to the study given in [18]. During training, the ANFIS network learns to map the coordinates of the ankle joint $x$ and $y$ to the joint angles $\theta_{1}$ and $\theta_{2}$. The trained ANFIS network is then used as a part of a larger control system to control the robotic arm. The ANFIS network, which has a multilayer feed forward structure, is shown in Fig. 7. In this structure each node performs a particular function on incoming signals and produces the required outputs. Note that the circle indicates a fixed node whereas a square indicates an adaptive node in which the parameters are changing during training.

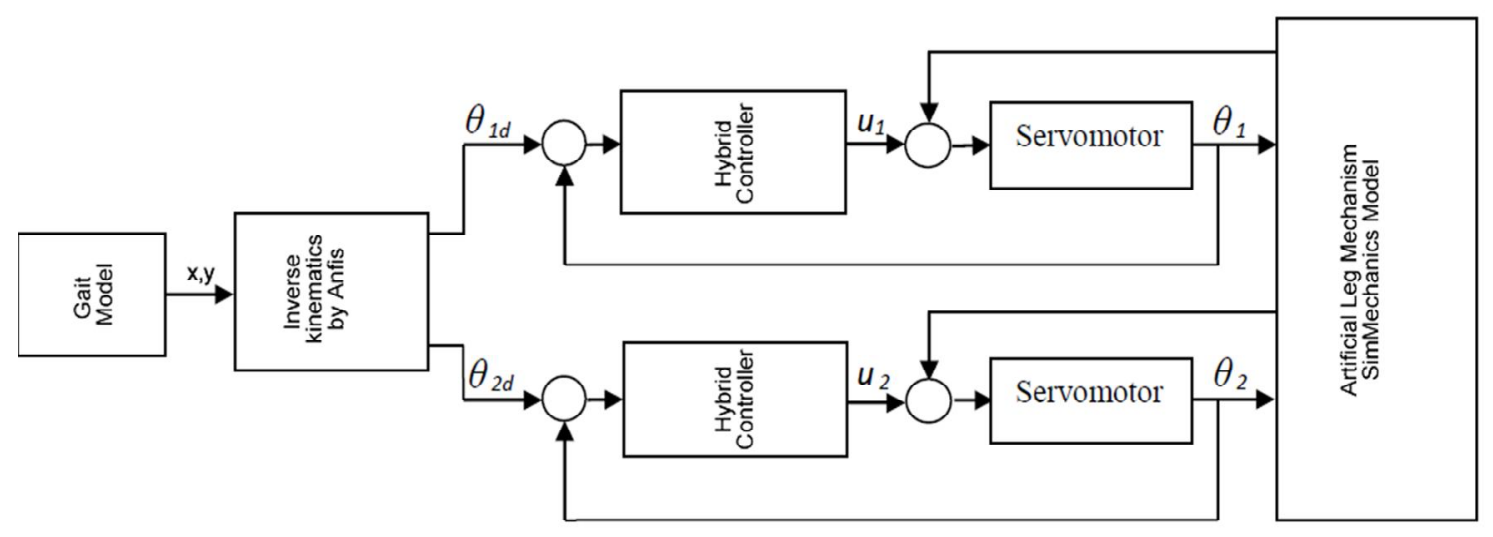

Figure 6. Overall control block diagram of the system. 


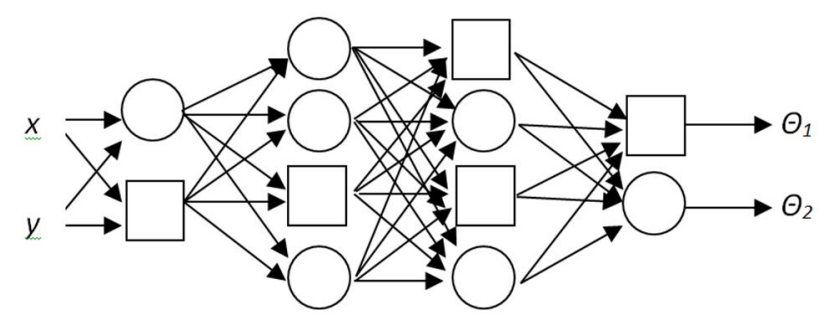

Figure 7. General adaptive neural network structure with two inputs and two outputs.

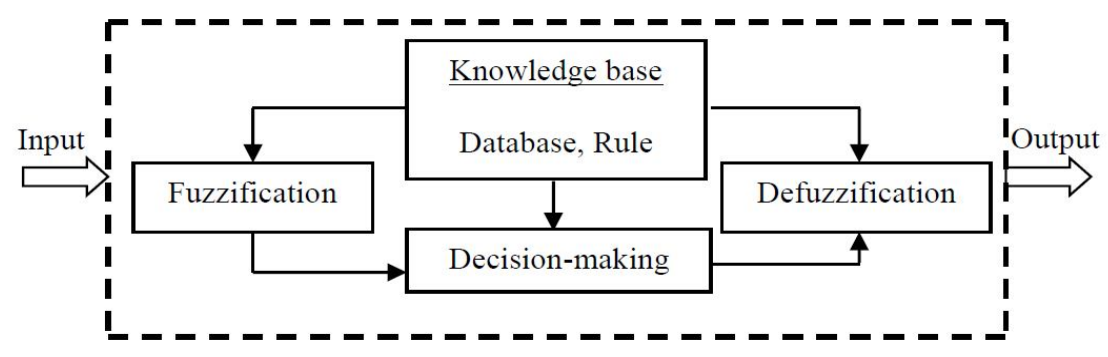

Figure 8. Fuzzy inference system.

The simple mathematical description of an artificialneural model is given as

$\theta_{i}(k)=F\left(\sum_{i=0}^{m} w_{i}(k) \cdot x_{i}(k)+b\right), i=1,2$

where $x_{i}(k)$ are the input values in discrete time $k$, and $i$ ranges from 0 to $m . w_{i}(k)$ are the weight values in discrete time $k$ where $i$ ranges from 0 to $m$ and $b$ is the bias. $F$ is a transfer function and $\theta_{i}(k)$ is the output value in discrete time $k$.

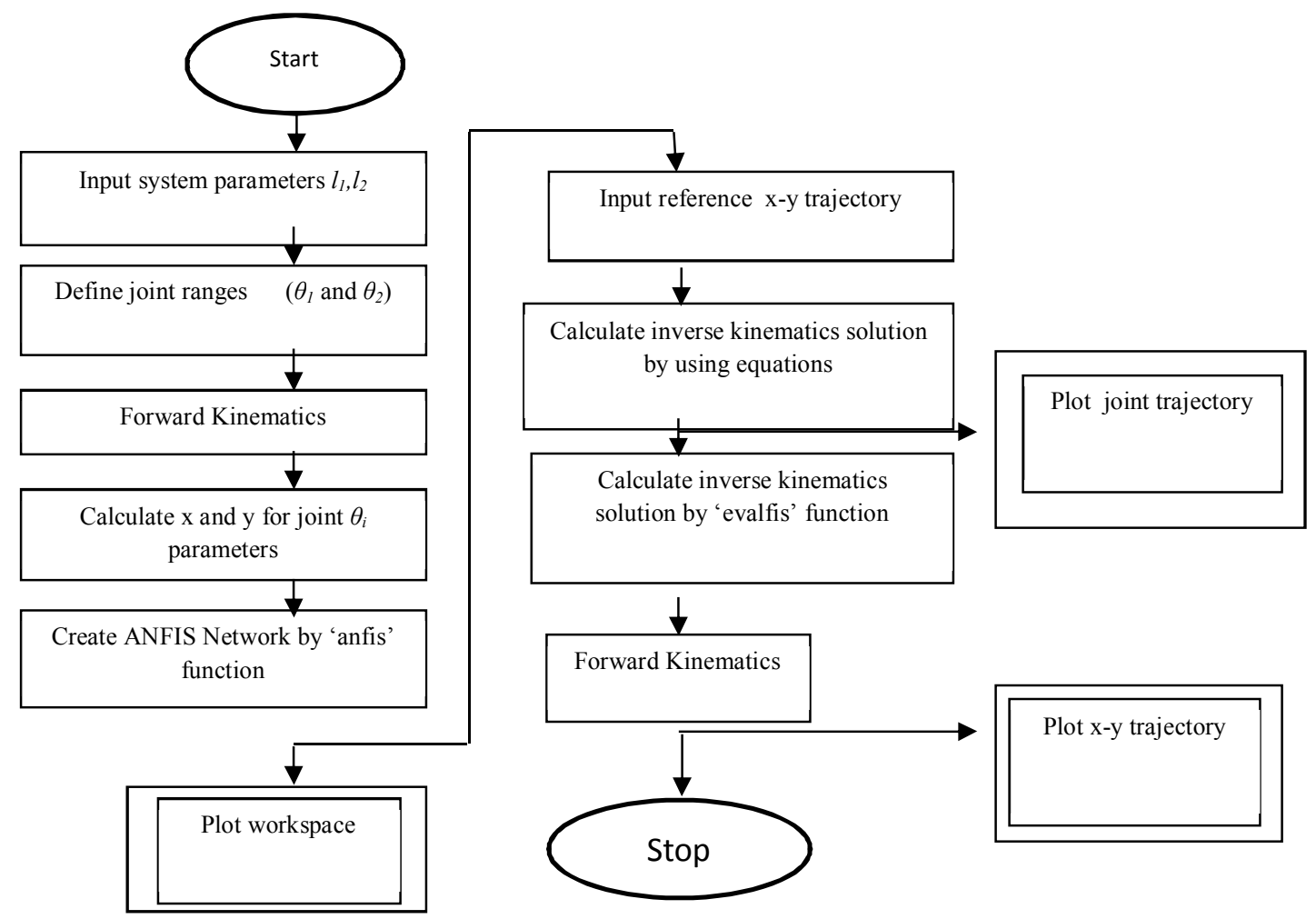

Figure 9. Flowchart of the program. 


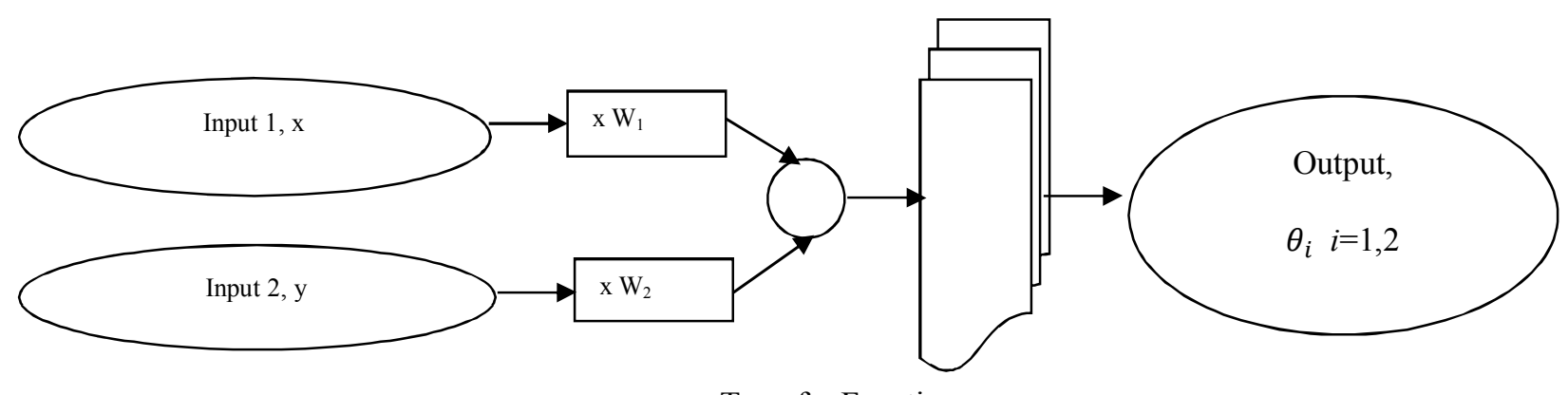

Transfer Function

Figure 10. Working principle of ANFIS for inverse kinematics.

During training, the ANFIS network learns to map the coordinates $x$ and $y$ to the angles $\theta_{1}$ and $\theta_{2}$. The trained ANFIS network is then used as

a part of a larger control system to control the robotic arm. Knowing the desired location of the robotic arm, the control system uses the trained ANFIS network to determine the angular positions of the joints. Subsequently, the controller applies the desired force at each joint of the robotic arm in order to move it to the desired location.

\section{Simulations}

\subsection{ANFIS model of the system}

The inverse kinematic calculations are achieved by the embedded Matlab file designed according to the flow diagram shown in Fig. 9. Here, the basic idea is the application of three basic rules of neural networks which are multiplication, summation and activation. The inputs to the system are weighted so that every input value is multiplied by individual weights before getting summed at the middle section.

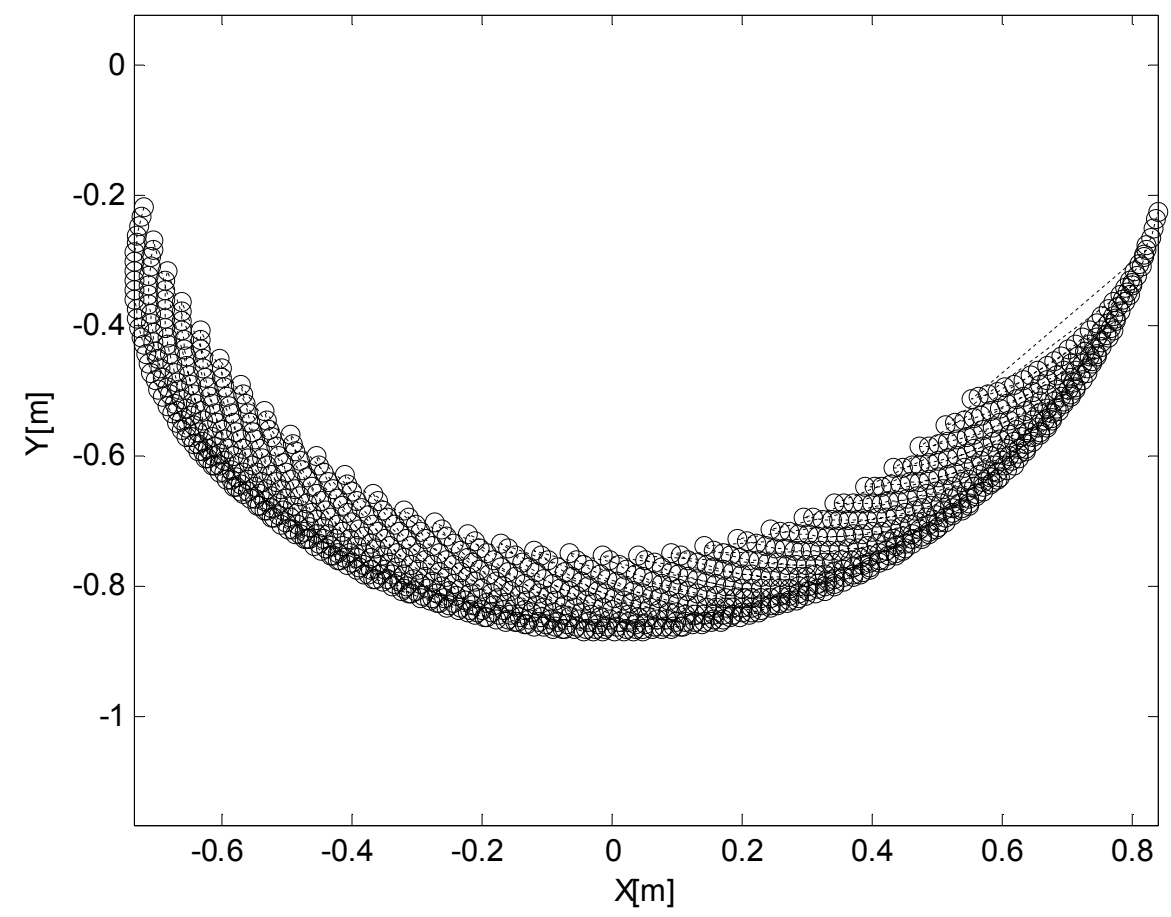

Figure 11. Workspace including the ankle motion during walking for $\theta_{1} 3.92-6.02$ rad. and for $\theta_{2} 5.23-6.28 \mathrm{rad}$. 


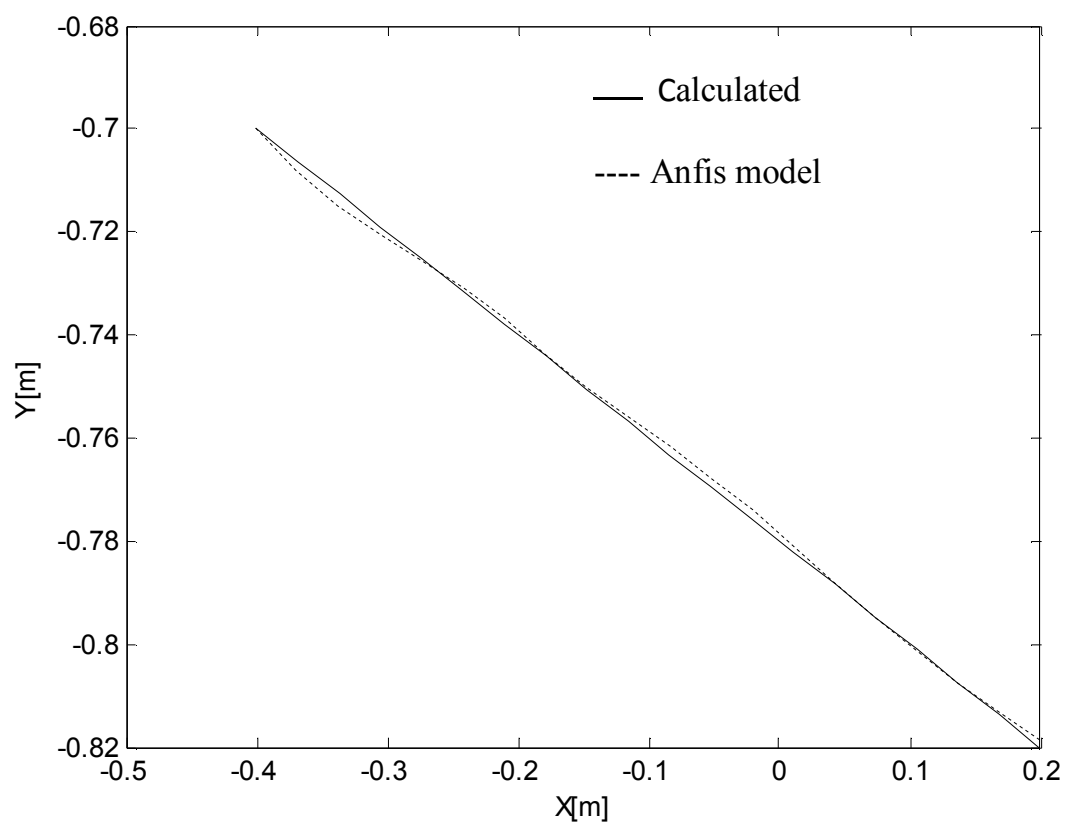

Figure 12. ANFIS model response vs. calculated trajectory for a linear trajectory.

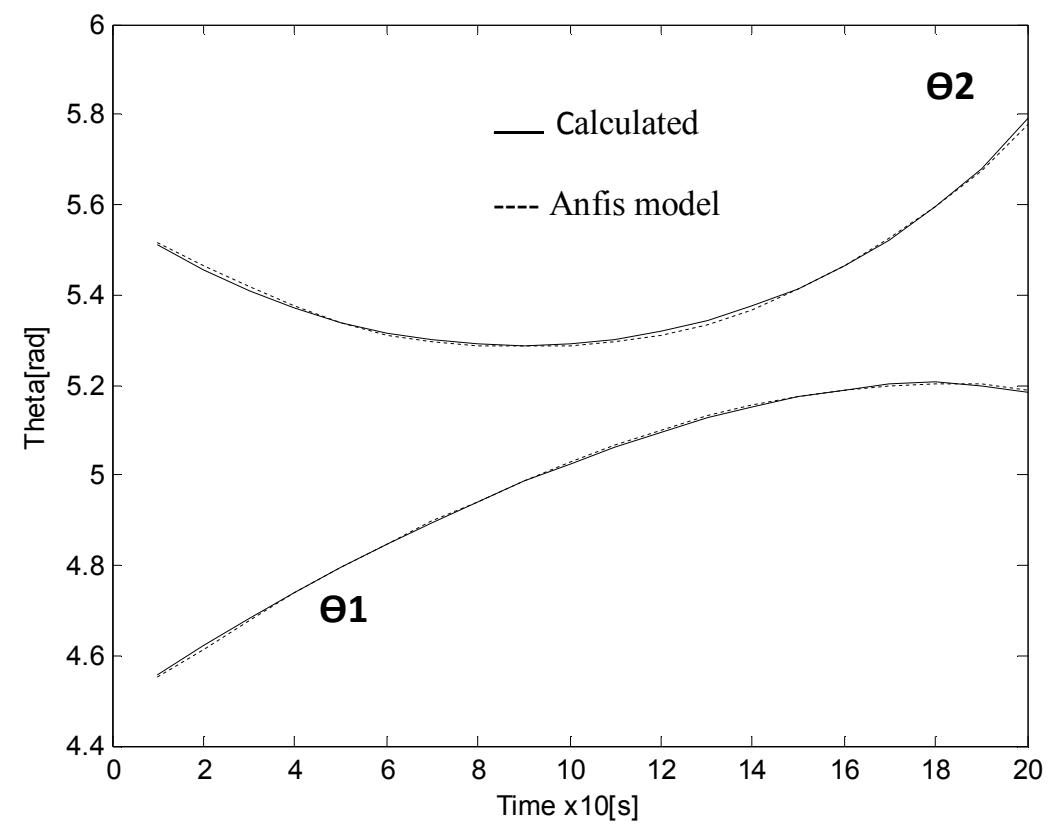

Figure 13. ANFIS model response vs. calculated trajectory for joint 1and joint 2. 


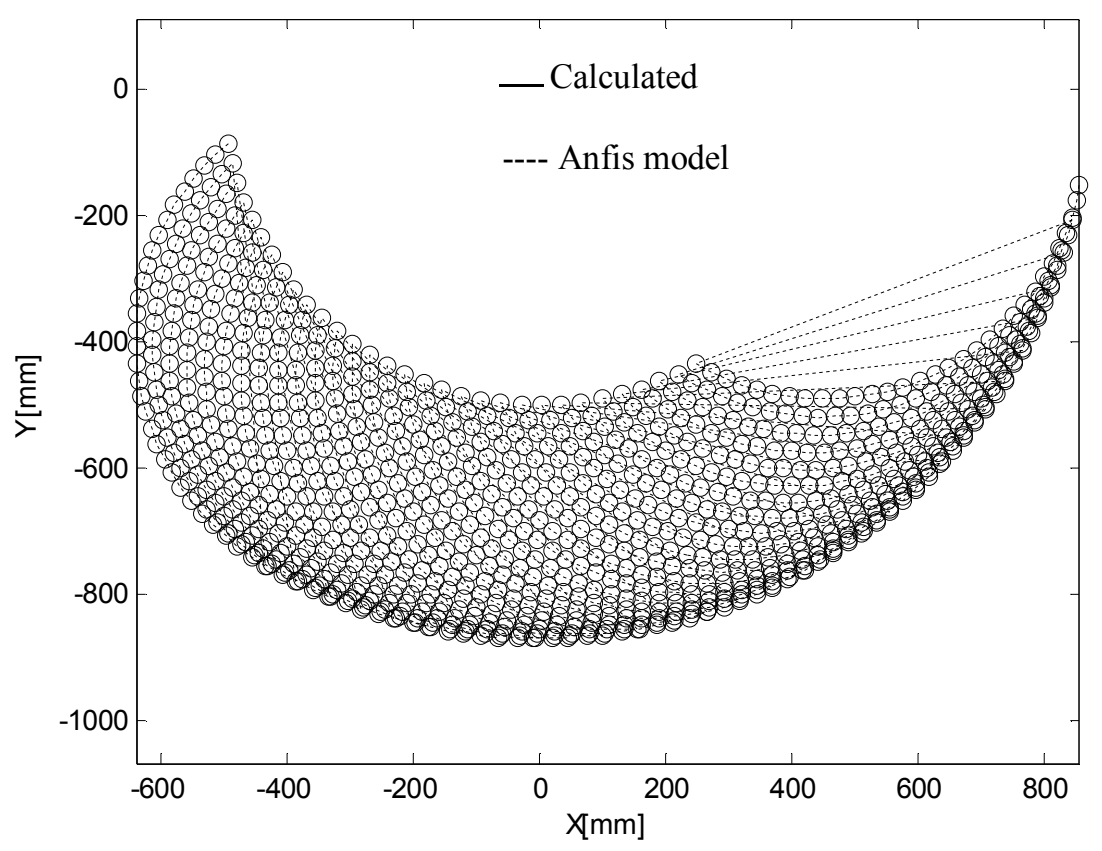

Figure 14. Workspace generated by ANFIS for gait model.

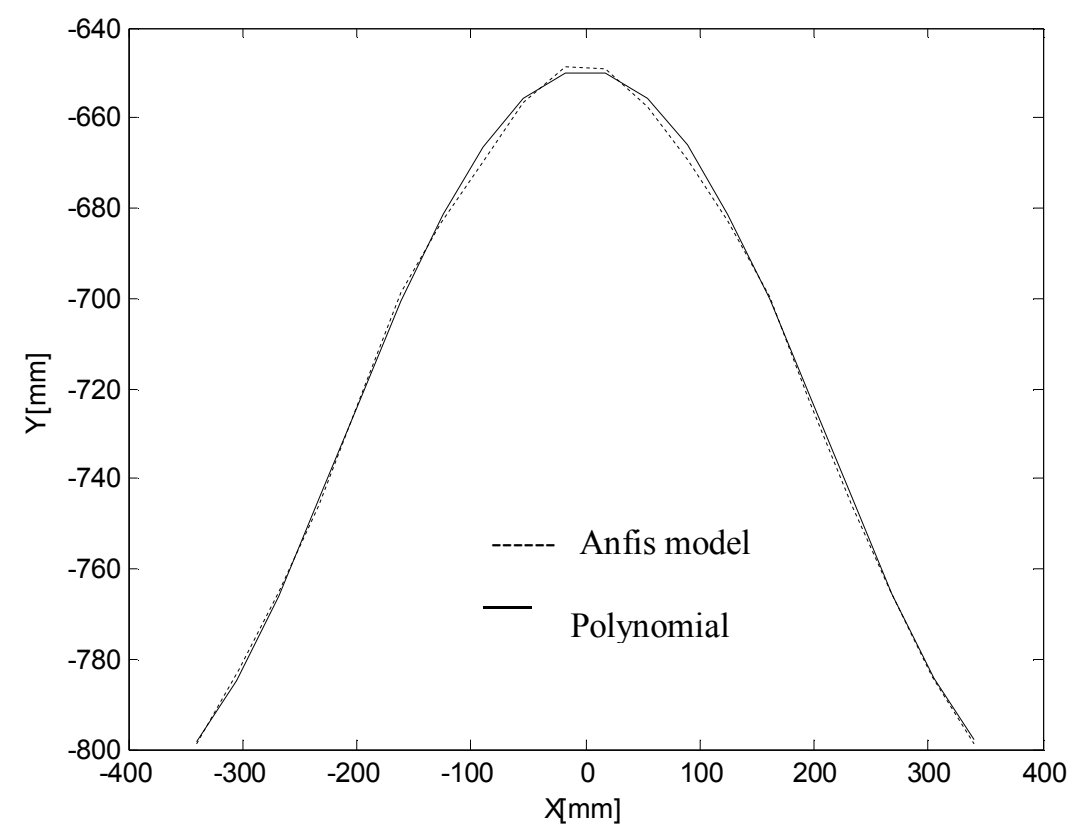

Figure 15. The calculated ankle joint Cartesian trajectory versus ANFIS model.

The output of the system is the sum of previously weighted inputs and bias introduced to an activation function known as transfer functions [19]. The inverse kinematic solution of the artificial leg mechanism using ANFIS is shown in Fig. 10. The thigh and the crus lengths of the assumed leg are $460 \mathrm{~mm}$ and 410 $\mathrm{mm}$, respectively. After introducing these values into the embedded Matlab file, the produced possible workspace for the ankle joint is assumed leg are $460 \mathrm{~mm}$ and $410 \mathrm{~mm}$, respectively. After introducing these values into the embedded Matlab file, the produced possible workspace for the ankle joint is depicted in Fig. 11. 
For testing the developed model, a linear trajectory is assumed for the endpoint of the leg mechanism. The results of the calculation and the ANFIS model are given in for a linear trajectory in Fig. 12. The corresponding hip and knee angular motions are obtained by calculating through the inverse kinematic relations and also ANFIS model provided and given in Fig. 13.

\subsection{Gait modeling}

After verifying the designed ANFIS model, the workspace necessary for ankle joint motion is generated as in Fig. 14. It's assumed for the gait model that the trajectory of the end point of the leg mechanism will be within this envelope. The approximated $5^{\text {th }}$ degree polynomial trajectory and also the ANFIS response for the same trajectory are given in Fig. 15.

The corresponding hip and knee angular motions are obtained by calculating through the inverse kinematic relations and also ANFIS model provided and given in Fig. 16. Both of the graphs show that the ANFIS model response is very close to calculated exact values.

\subsection{The architecture of the hybrid controller design}

Two separate PD plus Fuzzy hybrid controllers are designed and applied to the servomotors with specifications given in Table 1. The selected servomotors will drive a specially considered mechanism that is modeled with SimMechanics. As can be seen in Fig. 6, each hybrid position controller drives the servomotors to perform a gait motion whose block diagrams are given in Fig. 18. The detail of the controller is given in Fig. 17 where $K$ is the normalization constant of the output of the FLC and $E(s)$ is the error $\theta_{d}(s)-\theta_{m}(s)$ between the desired angular position of the joint and the output of the gearbox. The working principle of the FLC is shown in Fig. 8.

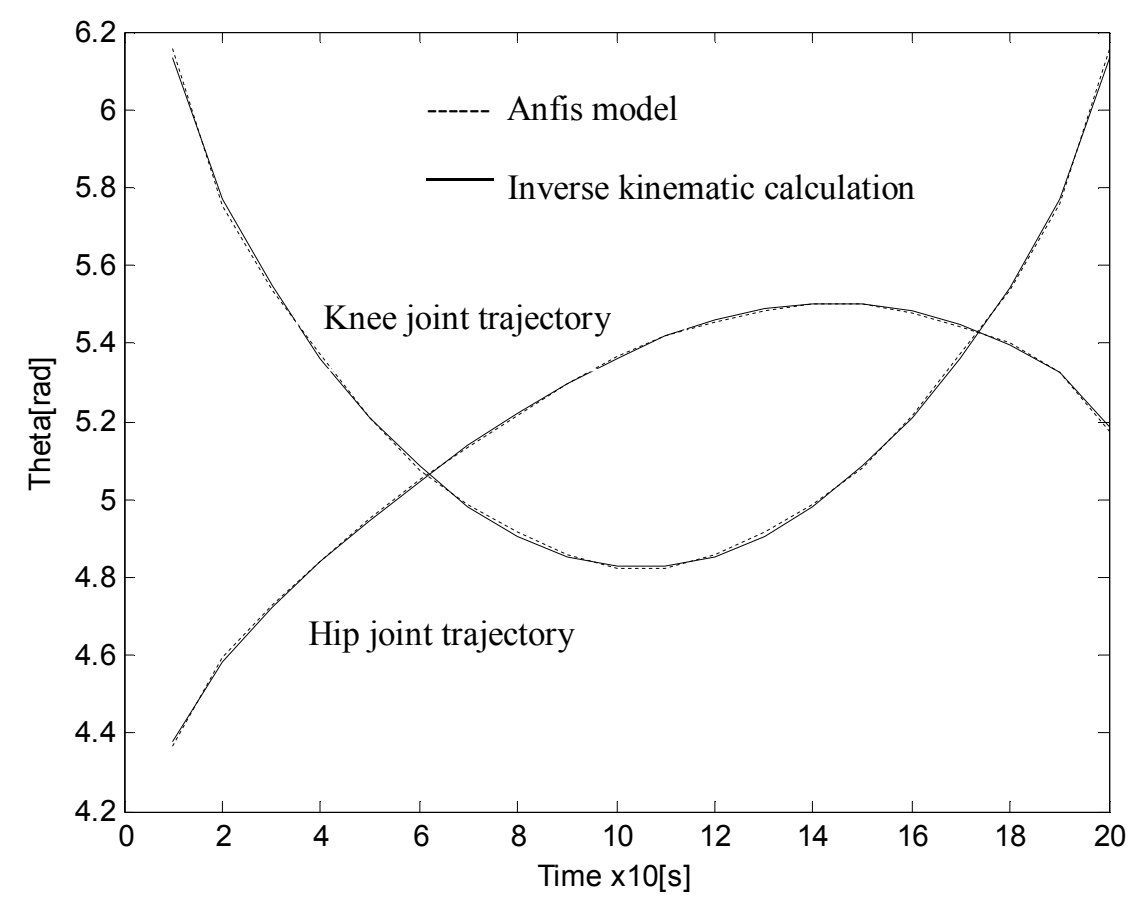

Figure 16. The calculated hip and knee joint trajectory versus ANFIS model. 


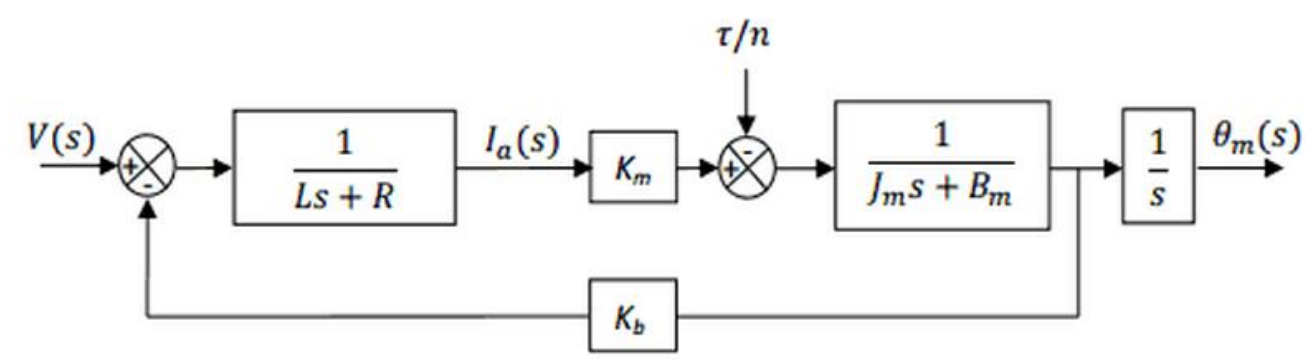

Figure 18. Servomotor model.

Proportional and derivative gain constants are determined by tuning the system, and the values belonging to both controllers are given in Table 3. The fuzzy logic part of the controller is desired in order to implement the integral gain component of the controller with a dynamic response. The actual inputs to the FLC system are the position error $(e)$ and the derivative of the error $(d e)$. The actual output is the control signal $(u)$. The inputs and the output of the FLC must be weighted by some error scaling factors $[20,21]$.

The applied fuzzy rules are given in Table 4 and the input membership functions are shown in Fig. 19, respectively. The Mamdani inference system is used to train the membership functions and produce the output for the given inputs.

Table 3. PD parameters used in the simulations.

\begin{tabular}{|l|c|c|}
\cline { 2 - 3 } \multicolumn{1}{c|}{} & $\begin{array}{c}\text { Hip joint } \\
\text { Servomotor }\end{array}$ & $\begin{array}{c}\text { Knee joint } \\
\text { Servomotor }\end{array}$ \\
\hline Proportional gain & 6172 & 21751 \\
\hline Derivative gain & 75 & 14 \\
\hline Derivative gain filter coefficient (N) & 223 & 358 \\
\hline
\end{tabular}

Table 4. A typical rule base for FLC.

\begin{tabular}{|l|l|l|l|l|l|}
\hline elde & NB & NS & Z & PS & PB \\
\hline NB & VS & M & VB & M & VS \\
\hline NS & VS & M & B & M & VS \\
\hline Z & VS & S & M & S & VS \\
\hline PS & VS & M & B & M & VS \\
\hline PB & VS & M & VB & M & VS \\
\hline
\end{tabular}


The explanation to the abbreviations outlined in Table 4 is provided as follows. NB: Negative big, NS: Negative small, Z: Zero, PS: Positive small, PB: Positive big, VS: Very small, M: Medium, S: Small, B: Big, VB: Very big.
Utilizing SimMechanics Toolbox of Matlab in Simulink, the simulation model to control and visualize the leg motion is developed. The corresponding ankle joint trajectory is given in Fig. 20.
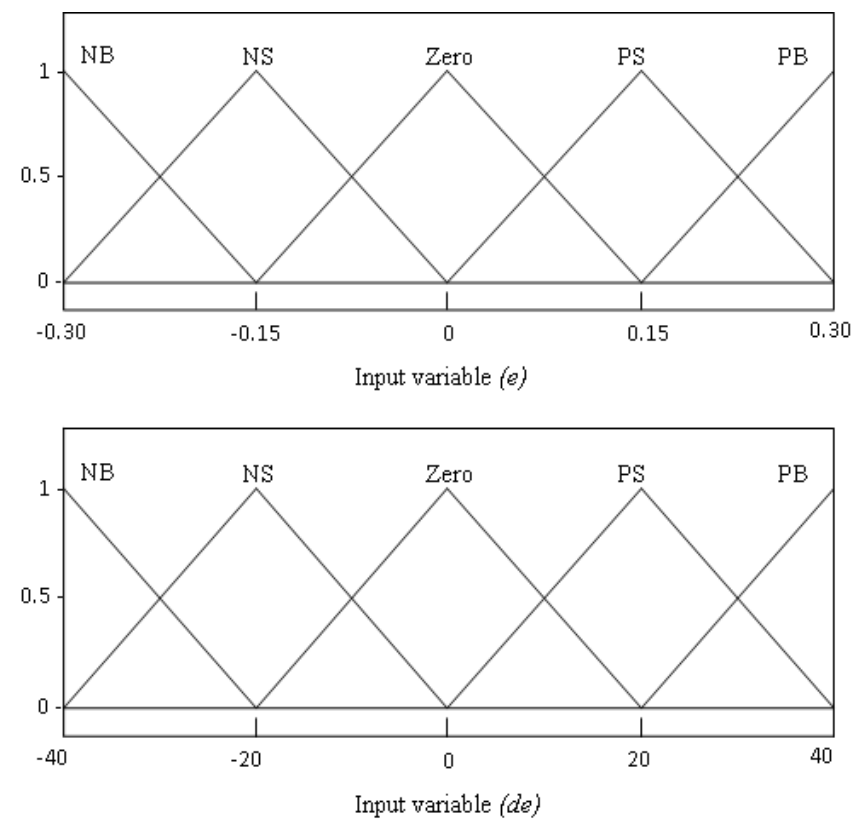

Figure 19. Membership functions of the first servomotor for inputs $e$ and $d e$.

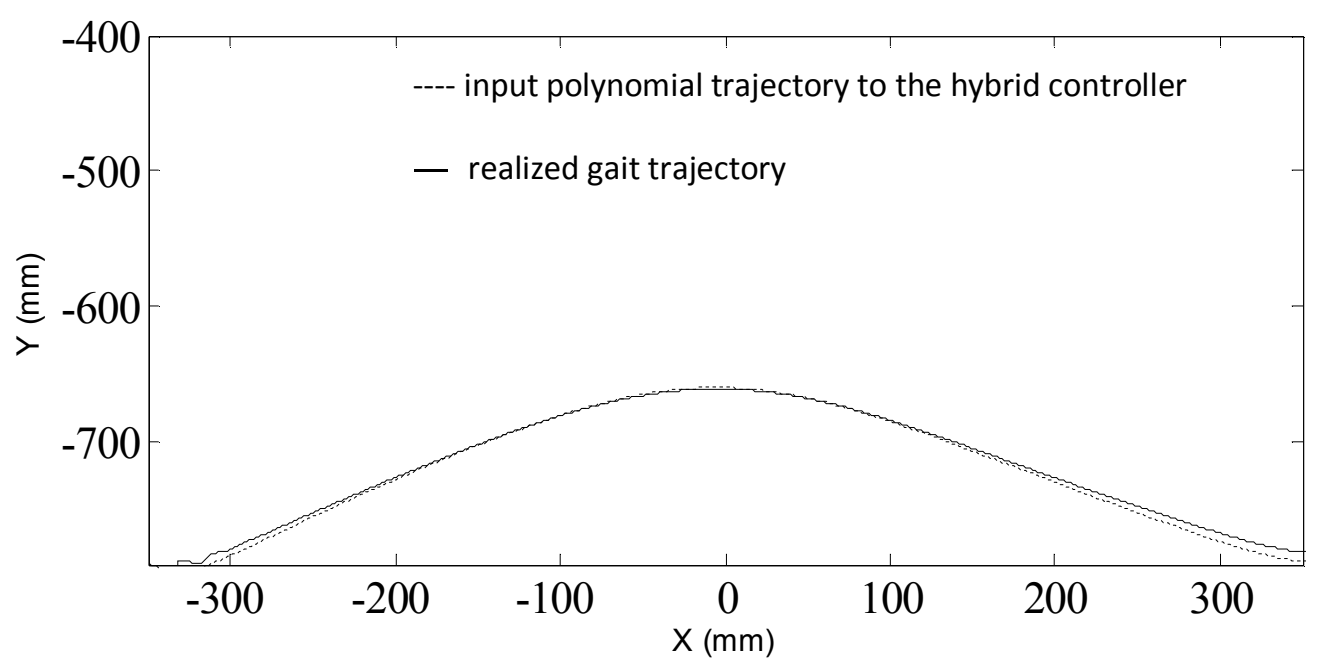

Figure 20. Response of the overall system to Anfis generated joint trajectories at ankle joint.

\section{Conclusion}

In this study, the ANFIS approach for the inverse kinematics problem is applied to generate joint trajectories. A hybrid type PD plus FLC controller is then applied for precise Cartesian trajectory tracking of the ankle joint of an artificial leg mechanism for the imposed gait model. The simulation results show that the system delivers acceptable results where the 
performance of the mechanism is improved considerably. The dynamic and coupling effects are added to the control system by means of the sensors of the SimMechanics model. Although the application of ANFIS to this mechanism with two degrees of freedom may be considered as simple, it rather constitutes a foundation for the analysis and control of mechanisms with higher degrees of freedom. In the first part of the paper, the exact solution of the kinematic equations is compared with the output of the ANFIS system. It is shown that the system behaves nicely for a linear trajectory of the ankle joint defined in the workspace of the gait model. The human gait is approximated as a $5^{\text {th }}$ order polynomial in this paper using the experimental data obtained through measurements. By using the previously trained ANFIS model, the corresponding joint angular motions for the Cartesian trajectory are generated and introduced as reference positions to the control system. The addition of the FLC in parallel to the PD controller enables the system to behave like a PID controlled system with adaptive integral gains which are readjusted based on the changing dynamics of the overall mechanism. Running the system with the smallest steady state error was the main objective of this controller model where the validity of such approach was demonstrated though simulations.

\section{REFERENCES}

[1] Zlajpah L. Simulation in Robotics. Mathematics and Computers in Simulation. 79879-897, 2008.

[2] Jazar RN. Theory of Applied Robotics. Springer;2006.

[3] Spong MW. Seth Hutchinson, M. Vidyasagar. Robot Modelling and Control. John Wiley and Sons; 2006.

[4] Qiao S, Liao Q, Wei S, Su HJ. Inverse kinematic analysis of the general $6 \mathrm{R}$ serial manipulators, Mechanism and Machine Theory 2010; 45:193-199.

[5] Manocha D, Canny JF. Efficient Inverse Kinematics for General 6R Manipulators, IEEE Transactions on Robotics and Automation 1994;Vol. 10, No. 5, 1994.

[6] Williams RL. II, Inverse Kinematics and Singularities of Manipulators with offset Wrist. IASTED International Journal of Robotics and Automation 1999;14-1:1-8.

[7] Howard DW and Zilouchian A. Application of Fuzzy Logic for the Solution of Inverse Kinematics and Hierarchical Controls of Robotic Manipulators. Journal of Intelligent and Robotic Systems 1998; 23:217-247.

[8] Jang JR. ANFIS: Adaptive-Network-Based Fuzzy Inference System. Man and Cybernetics 1993; 23- 3:665-685.

[9] Er MJ, Low CB, Nah KH, Lim MH, Yong NS. Real- time implementation of a dynamic fuzzy neural networks controller for a SCARA. Microprocessors and Microsystems 2002; 26:449-461.

[10] Popescu MC, Borcosi I, Olaru O, Popescu $\mathrm{L}$ and Grofu F. The simulation hybrid fuzzy control of a scara robot. WSEAS Transactions on Systems and Control 2008; 3:105-114.

[11] Doke J, Donelan MJ and Kuo AD. Mechanics and energetics of swinging the human leg.The Journal of Experimental Biology 2005;208:439-445.

[12] Aritan S, Cilli M, Amca AM. HUBAG: 3 Dimensional motion analysis software. Sport Science Journal (in Turkish) 2010;211:30-36. ISSN : 1300-3119

[13] Siciliano B, Khatip O. Handbook of Robotics. Springer, 2008.

[14] Ogata K. Modern Control Engineering. Prentice-Hall, Inc., 1997. 
[15] Yang Y, Zhou C. Robust Adaptive Fuzzy Control for Permanent Magnet Synchronous Servomotor Drives. International Journal of Intelligent Systems 2005; 20:153-171.

[16] Shieh MY and Li TS. Design and implementation of integrated fuzzy logic controller for a servomotor system. Mechatronics 1998;8:217-240.

[17] Çunkas M and Aydoğdu O. Realization of fuzzy Logic controlled brushless dc motor drives using Matlab/Simulink. Mathematical and Computational Applications 2010;152:218-229.

[18] Ankarali A. ANFIS inverse kinematics and precise trajectory tracking of a dual arm robot. Proceedings of the 2012 International Conference on Modelling, Simulation and Visualization Methods. 270-274, WORLDCOMP'12, July16-19, Las Vegas Nevada, USA, 2012

[19] Suzuki K. Artificial Neural NetworksMethodological Advances and Biomedical Applications. InTech 2011.
[20] Passino KM, Yurkovich S. Fuzzy Control. Addison-Wesley Longman;1998.

[21] Genc HM, Yesil E, Eksin I, Guzelkaya $\mathrm{M}$, Tekin OA. A rule base modification scheme in fuzzy controllers for time-delay systems. Expert Systems with Applications 2009;36:8476-8486. 